ANÁLISE DA RELAÇÃO ENTRE A INFRAESTRUTURA FÍSICA E AS REDES DE RELACIONAMENTOS INTEREMPRESARIAIS NOS PARQUES TECNOLÓGICOS BRASILEIROS

\title{
ANALYSIS OF THE RELATIONSHIP BETWEEN PHYSICAL INFRASTRUCTURE AND THE NETWORKS OF INTER-RELATIONSHIPS IN THE BRAZILIAN TECHNOLOGICAL PARKS
}

\section{ANÁLISIS DE LA RELACIÓN ENTRE LA INFRAESTRUCTURA FÍSICA Y LAS REDES DE RELACIONES ENTRE NEGOCIOS EN LOS PARQUES TECNOLÓGICOS BRASILEÑOS.}

Lucas Bonacina Rolda

Doutor em Administração de Empresas (PUCRS/UCS). Mestre em Administração de Empresas (PUCRS). Professor e Coordenador do Curso de Administração - Linha de Formação em Inovação e Empreendedorismo da Escola de Negócios da PUCRS

lucas.roldan@pucrs.br

http://orcid.org/0000-0002-3881-3918

\section{Alexandre Bom Veiga}

Graduado em Administração pela PUCRS. Técnico Administrativo na Pontifícia Universidade Católica do Rio Grande do Sul - PUCRS e no parque Tecnológico da PUCRS - TECNOPUC

alexandre.veiga@pucrs.br

Peter Bent Hansen

Doutorado em Engenharia de Produção pela Universidade Federal do Rio Grande do Sul . Professor e pesquisador do Programa de Pós-Graduação em Administração - PPGAd, da Pontifícia Universidade Católica do Rio Grande do Sul - PUCRS e professor nas Faculdades de Administração e Engenharia de Produção da PUCRS.

hansen.peter.57@gmail.com

https://orcid.org/0000-0003-2361-3574

Fernando Fantoni Bencke

Graduado em Administração pela Unochapecó, Licenciado em Filosofia pela Unochapecó, Professor e Pesquisador do Mestrado Profissional em Administração e Doutorado em Administração da Universidade do Oeste de Santa Catarina - UNOESC

fernando.bencke@unoesc.edu.br https://orcid.org/0000-0001-9721-3173

\section{Editor Científico: José Edson Lara}

Organização Comitê Científico

Double Blind Review pelo SEER/OJS

Recebido em 24.08.2019

Aprovado em 03.08.2020 


\title{
Resumo
}

Objetivo: este artigo tem como objetivo analisar a relação entre a adequação das infraestruturas físicas dos parques tecnológicos e as interações nas redes de relacionamentos entre as empresas residentes, e entre as empresas e as universidades.

Metodologia/abordagem: um modelo teórico foi elaborado e testado quantitativamente por meio de modelagem de equações estruturais, analisado a partir de uma amostra válida de 189 empresas residentes em parques tecnológicos brasileiros.

Originalidade/Relevância: estudar as infraestruturas físicas dos parques tecnológicos traz importantes inputs para o desenvolvimento de políticas de apoio à inovação, já que diversas iniciativas de promoção de parques tecnológicas vêm sendo desenvolvidas nos últimos anos.

Principais resultados: foram encontradas relações significativas sobre a influência da infraestrutura física dos parques tecnológicos sobre as interações entre as empresas residentes e entre as empresas e universidades, e a importância de uma infraestrutura física adequada para gerar interação à inovação.

Contribuições teóricas: este estudo ajudou a demonstrar que não basta a existência de infraestruturas físicas nos parques tecnológicos; estas precisam ser adequadas ao ambiente para que gerem interações.

Contribuições para a gestão: evidenciou-se que os governos precisam fomentar não apenas a criação de parques tecnológicos, mas sim de parques tecnológicos que possuam infraestruturas físicas capazes de proporcionar interação entre os atores do sistema de inovação

Palavras-chave: Parques Tecnológicos; Infraestrutura Física; Interações; Redes de Relacionamentos.

\begin{abstract}
Objective: this article aims to analyze the relationship between the adequacy of the physical infrastructures of technology parks and the interactions in the networks of relationships between resident companies, and between companies and universities.
\end{abstract}

Methodology / approach: a theoretical model was elaborated and tested quantitatively through structural equation modeling, analyzed from a valid sample of 189 companies residing in Brazilian technology parks.

Originality / Relevance: studying the physical infrastructures of technology parks brings important inputs for the development of policies to support innovation, as several initiatives to promote technology parks have been developed in recent years.

Main results: significant relationships were found regarding the influence of the physical infrastructure of technology parks on the interactions between resident companies and between companies and universities, and the importance of an adequate physical infrastructure to generate interaction with innovation.

Theoretical contributions: this study helped to demonstrate that the existence of physical infrastructures in technology parks is not enough; these need to be adapted to the environment in order to generate interactions. 
Contributions to management: it became evident that governments need to encourage not only the creation of technology parks, but technology parks that have physical infrastructures capable of providing interaction between the actors of the innovation system

Keywords: Technology Parks; Physical Infrastructure; Interactions; Relationship Networks.

\section{Resumen}

Objetivo: este artículo tiene como objetivo analizar la relación entre la adecuación de la infraestructura física de los parques tecnológicos y las interacciones en las redes de relaciones entre empresas residentes, y entre empresas y universidades.

Metodología / enfoque: se elaboró y probó cuantitativamente un modelo teórico mediante el modelado de ecuaciones estructurales, analizado a partir de una muestra válida de 189 empresas que residen en parques tecnológicos brasileños.

Originalidad / relevancia: el estudio de las infraestructuras físicas de los parques tecnológicos aporta importantes aportes para el desarrollo de políticas de apoyo a la innovación, ya que en los últimos años se han desarrollado varias iniciativas para promover los parques tecnológicos.

Resultados principales: se encontraron relaciones significativas con respecto a la influencia de la infraestructura física de los parques tecnológicos en las interacciones entre empresas residentes y entre empresas y universidades, y la importancia de una infraestructura física adecuada para generar interacción con la innovación.

Contribuciones teóricas: este estudio ayudó a demostrar que la existencia de infraestructuras físicas en los parques tecnológicos no es suficiente; estos deben adaptarse al entorno para generar interacciones.

Contribuciones a la gestión: se hizo evidente que los gobiernos deben alentar no solo la creación de parques tecnológicos, sino también parques tecnológicos que tienen infraestructuras físicas capaces de proporcionar interacción entre los actores del sistema de innovación.

Palabras clave: Parques tecnológicos; Infraestructura física; Interacciones; Redes de relaciones.

\section{Introdução}

O mundo corporativo tem passado por constantes mudanças, devido à alta competitividade e ao dinamismo do ambiente empresarial (Ferreira, Soria, \& Closs, 2012). Estas transformações ocorrem em razão da relevância dada ao conhecimento (Wolfarth, 2004), estimulado pela globalização e pelo desenvolvimento tecnológico em constante evolução (Ferreira et al., 2012). Devido à busca constante por vantagens competitivas, a inovação se tornou um tema importante para as empresas (Sereda, Dietrich, \& Hermann, 2015). Em virtude 
desse contexto, cresceu a necessidade da interação entre as organizações, que constitui uma maneira de gerar benefícios mútuos (Ferreira et al., 2012), tornando-se importante para a inovação e o crescimento econômico fundamentado no conhecimento (Etzkowitz, 2013).

Alguns estudos indicam que a proximidade geográfica incita a formação de relações espaciais (próximas) entre as empresas e as empresas e as universidades, definindo este contexto como rede de relacionamento capaz de estimular a interação (Cantù, 2010; MolinaMorales \& Martínez-Cháfer, 2016). Quanto mais frequente fora interação, mais as empresas compreendem os benefícios das redes de relacionamento, propiciando a colaboração em projetos, consultorias e contatos informais (Soetanto \& Jack, 2013).

Uma das iniciativas que surgiram para fomentar a interação entre empresas é constituída pelos Parques Tecnológicos. O primeiro parque surgiu no ano de 1951, nos Estados Unidos, quando a Universidade de Stanford criou o chamado Stanford Industrial Park, com o propósito de abrigar empresas dispostas a trabalhar em conjunto com a academia (Spolidoro \& Audy, 2008). Essas iniciativas abriram caminho para uma série de empreendimentos similares concebidos ao redor do mundo nos anos seguintes (Bellavista \& Sanz, 2009; Spolidoro \& Audy, 2008).

A iniciativa dos parques tecnológicos começou a ser tratada no Brasil em 1984, devido a um programa de governo criado para apoiar esta iniciativa (ABDI \& ANPROTEC, 2008). No ano de 2000 a iniciativa passou a fazer parte dos planos do Governo Federal para promoção do desenvolvimento tecnológico e econômico do país (Vedovello, Judice, \& Maculan, 2006).

Neste estudo, os parques tecnológicos serão tratados genericamente, e independente do conceito atribuído a eles (parque tecnológico, parque de pesquisa, parque científico e parque empresarial). Eles têm como objetivo a disseminação de novas tecnologias, a interação (empresas, universidade, governo e sociedade), a constituição de novas empresas e a criação de empregos (Vedovello et al., 2006). Estes empreendimentos também se caracterizam pelo incentivo à inovação, competitividade e a disseminação do conhecimento entre os seus parceiros (ABDI \& ANPROTEC, 2008; Figlioli \& Porto, 2012), ficando implícito em seu conceito a criação de estruturas físicas capazes de propiciar o desenvolvimento (Gower\& Harris, 1996) e contribuir para o crescimento da região onde estão inseridos (Baldoni \& Furtado, 2014; Pradella, 2013).

A infraestrutura física se torna uma ferramenta importante à interação nas redes de relacionamentos (Baldoni \& Furtado, 2014; Figlioli \& Porto, 2012; Gower \& Harris, 1996) e 
são considerados fatores críticos para o sucesso dos parques tecnológicos (Baldoni \& Furtado, 2014).

Segundo Etzkowitz e Zhou (2018) a infraestrutura física é considerada um fator facilitador para melhorar o desempenho da inovação em parques tecnológicos. Por outro lado, como um elemento isolado, não é suficiente, deve fazer parte de um conjunto amplo de interações envolvendo diferentes atores como a universidade, empresas e governo. A infraestrutura é gerada e utilizada de diferentes maneiras por parques no mundo, os autores citam os exemplos dos parques de Stanford nos Estados Unidos e Porto Digital em Recife, onde a infraestrutura foi criada como resultado de um processo interativo para a inovação

Devido à influência dos parques tecnológicos no estímulo à inovação, fomentada pela proximidade física dos atores envolvidos nesses ambientes (Vedovello et al., 2006), através das possibilidades de redes de relacionamentos entre os atores envolvidos (Cantù, 2010), este trabalho tem como objetivo analisar as relações entre a adequação das infraestruturas físicas e as interações nas redes de relacionamentos entre as empresas residentes e as outras empresas (EE) e, entre as empresas residentes com as universidades (UE) nos parques tecnológicos brasileiros. A finalidade da pesquisa é verificar se a adequação das infraestruturas físicas influencia positivamente as interações nas redes de relacionamentos EE e UE. Um modelo teórico foi elaborado e testado quantitativamente por meio de modelagem de equações estruturais, analisado a partir de uma amostra válida de 189 empresas residentes em parques tecnológicos brasileiros.

A próxima seção apresentará o referencial teórico utilizado no estudo juntamente com as hipóteses de pesquisa, após será demonstrada a metodologia utilizada, seguindo pelos resultados da pesquisa e, por fim, serão expostas as considerações finais do estudo.

\section{Referencial teórico e hipóteses de pesquisa}

Nesta seção são apresentados os levantamentos bibliográficos referentes às infraestruturas físicas dos parques tecnológicos, interações nas redes de relacionamento entre as empresas residentes, e entre as empresas e as universidades, assim como as hipóteses geradas para este estudo. 


\subsection{Infraestruturas físicas dos parques tecnológicos}

As infraestruturas físicas dos parques tecnológicos surgem como um dos componentes do processo de inovação, através da interação proporcionada pela proximidade entre as empresas do parque e as universidades (Minguillo, Tijssen, \& Thelwall, 2015; Vedovello et al., 2006). Este processo visa atender alguns dos objetivos destes empreendimentos, como o apoio à geração de novos negócios e crescimento das empresas (Gower \& Harris, 1996). Ferreira, Sória e Closs (2012) mencionam a importância destas estruturas de apoio e a criação de ambientes para estimular a interação entre a universidade e empresa. Cantú (2010), além de reforçar a importância das interações nas redes de relacionamentos entre universidades e empresas, ainda inclui as interações entre as empresas.

São diversas as infraestruturas físicas dos parques tecnológicos encontradas na literatura, com o propósito de fomentar a interação entre os atores deste ecossistema. Gower e Harris (1996) relacionam como infraestruturas físicas as instalações recreativas, instalações para formação empresarial, instalações para reuniões, palestras e conferências, cantinas e restaurantes, bar para reuniões informais e as instalações desportivas, como instalações e ambientes propícios ao desenvolvimento de interações. AABDI e a ANPROTEC (2008) referem-se à infraestrutura física como espaços destinados aos negócios, inovação, incubação, feiras, exposições e para criação de produtos.

Atrasas, Dias e Leite (2003) citam salas de reuniões, auditórios, salas de videoconferência, bancos, agências de viagens, hotéis e centros esportivos. Figlioli e Porto (2012) ressaltam a busca constante dos parques em adequar suas instalações, a fim de fomentar o processo de inovação através de um conjunto de infraestruturas físicas e citam como exemplo as infraestruturas básicas (terrenos, rede de água e esgoto, energia, telecomunicações, gás, rede viária e sinalização, áreas de estacionamento, passeios, tratamento e controle de resíduos, iluminação externa, infraestrutura de segurança e outros), os edifícios institucionais (instalações da gestão do parque, associações representativas, bancos de fomento e agências de desenvolvimento e de negócios), as infraestruturas tecnológicas (centros de P\&D, laboratórios de uso conjunto e outros), edifícios de negócios (locação ou venda para instalação de empresas, tais como incubadoras e centros empresariais) e as áreas verdes e sociais (destinadas ao convívio social, à prestação de serviços ao indivíduo, creches, restaurantes, cafés, parques ecológicos, local para prática de esporte, lazer, academia e outros) (Figlioli \& Porto, 2012). 
No relatório da ABDI e ANPROTEC (2008) observa-se que a qualidade da infraestrutura é um item recorrente nos modelos de parques tecnológicos de sucesso. Bellavista e Sanz (2009) mencionam que instalações de qualidade, em um espaço cuidadosamente projetado, é um elemento comum, considerado pela maioria dos parques na sua construção. Quando uma infraestrutura é bem definida, de qualidade, utilizada como um mecanismo de atração e ainda favorável à interação entre as empresas, faz emergir um ambiente tecnológico pré-existente (ABDI \& ANPROTEC, 2008), propício ao empreendedorismo, à inovação (Ferreira et al., 2012), ao desenvolvimento e ao crescimento (Gower \& Harris, 1996).

Assim, para a análise das infraestruturas físicas dos parques tecnológicos brasileiros, será utilizada a abordagem de Figlioli e Porto (2012), pois foi considerada a mais adequada, já que aborda as infraestruturas de uma forma sistêmica e para tal será trabalhada a adequação das mesmas conforme o Figura 1.

\begin{tabular}{|l|l|}
\hline $\begin{array}{l}\text { Infraestruturas } \\
\text { básicas }\end{array}$ & $\begin{array}{l}\text { Compostas por terrenos, rede de água, energia, telecomunicações (telefonia, rede de } \\
\text { fibra ótica, sistema wi-fietc.), esgoto, gás, rede viária e sinalização, áreas de } \\
\text { estacionamento, passeios, tratamento e controle de resíduos, equipamentos de } \\
\text { eliminação de resíduos, iluminação exterior, guaritas de segurança, entre outros. }\end{array}$ \\
\hline $\begin{array}{l}\text { Edifícios } \\
\text { institucionais }\end{array}$ & $\begin{array}{l}\text { Abrigam a organização gestora do parque, associações representativas das empresas, } \\
\text { bancos de fomento e agências de desenvolvimento. }\end{array}$ \\
\hline $\begin{array}{l}\text { Edifícios de } \\
\text { negócios }\end{array}$ & $\begin{array}{l}\text { Destinados à locação ou à venda para as empresas que queiram instalar-se no parque; } \\
\text { exemplos são incubadoras de empresas e centros empresariais (business centers). }\end{array}$ \\
\hline $\begin{array}{l}\text { Infraestruturas } \\
\text { tecnológicas }\end{array}$ & $\begin{array}{l}\text { Como centros tecnológicos de P\&D, laboratórios de pesquisa de uso conjunto, entre } \\
\text { outros. }\end{array}$ \\
\hline $\begin{array}{l}\text { Áreas verdes e } \\
\text { sociais }\end{array}$ & $\begin{array}{l}\text { Áreas destinadas ao convívio social e a serviços prestados ao indivíduo, que podem ser } \\
\text { compostas por áreas de esporte e lazer, academias, creches, restaurantes, cafés, parques } \\
\text { ecológicos etc. }\end{array}$ \\
\hline
\end{tabular}

Figura 1: Variáveis observadas da infraestrutura física

Fonte: Figlioli e Porto (2012, p. 291).

\subsection{Infraestrutra física e interação empresa-empresa em parques tecnológicos}

As empresas baseadas no conhecimento emergem das interações contínuas e recursivas, internas e externas à organização, que visam auxiliar na criação e na disseminação do conhecimento, no intuito de gerar inovação (Angeloni, 2002). A partilha do conhecimento adquirido entre as empresas pode ser constatada nos acordos e projetos conjuntos firmados entre elas, em busca de interesses mútuos (Bellavista \& Sanz, 2009; Soetanto \& Jack, 2013). As incubadoras existentes nos parques tecnológicos apresentam infraestruturas para apoiar o desenvolvimento das empresas e tem atraído um crescente interesse, por ser consideradas um 
instrumento eficaz no apoio ao crescimento de novas empresas intensas em tecnologia (Soetanto \& Jack, 2013).

Neste contexto, consideram-se as empresas como parte de uma rede capaz de influenciar e ser influenciada em razão da proximidade entre elas, tornando essa relação espacial (próxima) como ponte para unir as empresas ao seu ambiente (Araujo, Dubois, \& Gadde, 2003). A proposta de interação entre as empresas consolidadas e as startups instaladas nos parques tecnológicos é importante, pois existe a possibilidade de trocas de experiências, possibilitando que as empresas consolidadas, contribuam para sobrevivência das startups e vice-versa (Atrasas, Dias, \& Leite, 2003). Segundo Soetanto e Jack (2013), quanto maior assiduidade na interação entre as empresas, maior será a compreensão das suas necessidades, possibilitando a colaboração e o crescimento conjunto.

Cantù (2010) apresenta um estudo de caso da empresa Petroceramics (primeira spin-off gerada na Universidade de Milão - Itália) que residiu no POINT Italian Technological Poleeno Italian Kilometro Rosso Science Park que,por meio de relações espaciais (ou redes de relacionamentos), é possível se beneficiar da proximidade com outras empresas. O estudo de Soetanto e Jack (2013) trata sobre as empresas incubadas no Daresbury Science and Innovation Campus no Reino Unido e as suas relações com as redes de acesso aos recursos intangíveis e tangíveis. Estes autores apresentam em uma das suas recomendações finais a necessidade de construir um ambiente propício para o desenvolvimento das interações, buscando relações sólidas e mútuas. Sendo assim, pode-se considerar a seguinte hipótese.

Hipótese 1 - A adequação das infraestruturas físicas dos parques tecnológicos influencia positivamente as redes de relacionamentos das empresas residentes com outras empresas.

\subsection{Infraestrutura física e a interação universidade-empresa em parques tecnológicos}

O conhecimento científico tem sido considerado um insumo importante para o desenvolvimento econômico e, no modelo de interação universidade/empresa, ele se intensifica devido à relação de aprendizagem interativa e inovadora (Cantù, 2010; Ferreira et al., 2012). Os mecanismos (infraestruturas físicas) deste modelo são importantes, especialmente na transferência de tecnologia, podendo criar ligações com os mercados, aumentando a demanda por produtos e serviços (Audy, Cunha, \& Franco, 2002). 
A transferência de tecnologia baseia-se em um modelo que a universidade transfere o conhecimento às empresas, ou ainda, em um processo organizacional interno de troca, adaptação e aprendizagem de novas tecnologias, gerido e realizado em parceria com diversos atores (Cantù, 2010). As formas funcionais desta transferência podem ser potencializadas devido ao vínculo criado com o mercado, através das formas inovadoras de interação entre universidade e empresa (Audy et al., 2002). A tecnologia e o conhecimento passado dos produtores aos utilizadores fazem com que a interação se transforme em valor econômico às empresas (Cantù, 2010).

A interação entre estes atores tem como objetivo o fornecimento de diferentes recursos, a fim de combiná-los no estímulo à inovação (Cantù, 2010; Minguillo et al., 2015). Sendo assim, quanto mais frequente for a interação, maior será a colaboração (Soetanto \& Jack, 2013) e o processo torna-se bem sucedido, quando o resultado da pesquisa é útil e transferido efetivamente à empresa (Audy et al., 2002).

A partir desse contexto, a proximidade física e geográfica, baseada na cooperação, se torna importante no incentivo à interação, permitindo que os recursos adquiridos sirvam de apoio às atividades, promovendo a economia de tempo, acordos de interação e adaptações complexas (Cantù, 2010), demonstrando a importância das infraestruturas físicas como ferramentas de interação (Baldoni \& Furtado, 2014; Figlioli \& Porto, 2012; Gower \& Harris, 1996), tornando-se um fator crítico para o sucesso dos parques tecnológicos (Baldoni \& Furtado, 2014).

No estudo de caso de Cantù (2010) sobre a empresa Petroceramics, foram analisados os benefícios gerados em residir em parques tecnológicos, proporcionando cooperação e a sinergia das relações espaciais (ou redes de relacionamentos) com a Universidade de Milão e o Instituto de Processos de Ambiente Dinâmico. Estar situada em um parque proporcionou o apoio de infraestruturas físicas e serviços, tais como escritórios, salas de reuniões, laboratórios, serviço de patentes e a transferência de tecnologia para o desenvolvimento da empresa. As relações geradas nesses processos em conjunto com os demais atores, através da combinação dos recursos, resultou no desenvolvimento de inovações( Cantù, 2010).

O estudo de Audy, Cunha e Franco (2002) apresenta alguns modelos de interação: centros de pesquisa cooperativos, o parque e o polo tecnológico, a incubadora de empresas de base tecnológica e as joint ventures universidade/empresa como mecanismos de interação, buscando criar uma rede local de cooperação, interligando os atores envolvidos no processo. 
Soetanto e Jack (2013) expõem a relação da universidade e empresa com as atividades tangíveis e as relaciona com os níveis de inovação das empresas incubadas. Dessa forma, pode-se considerar a seguinte suposição.

Hipótese 2 - A adequação das infraestruturas físicas dos parques tecnológicos influencia positivamente nas redes de relacionamentos de empresas residentes com as universidades.

Conforme mencionado no levantamento teórico, as relações sugeridas entre as infraestruturas físicas e as interações (tratadas aqui como redes de relacionamentos) entre empresas (EE), e entre universidades e empresas (UE) nos parques tecnológicos são discutidas, e as variáveis geradas desta discussão propiciaram o desenvolvimento das hipóteses. O modelo teórico deste estudo é apresentado na Figura 2, se propõe que a adequação das infraestruturas físicas dos parques tecnológicos influência positivamente nas interações tipo EE e UE.

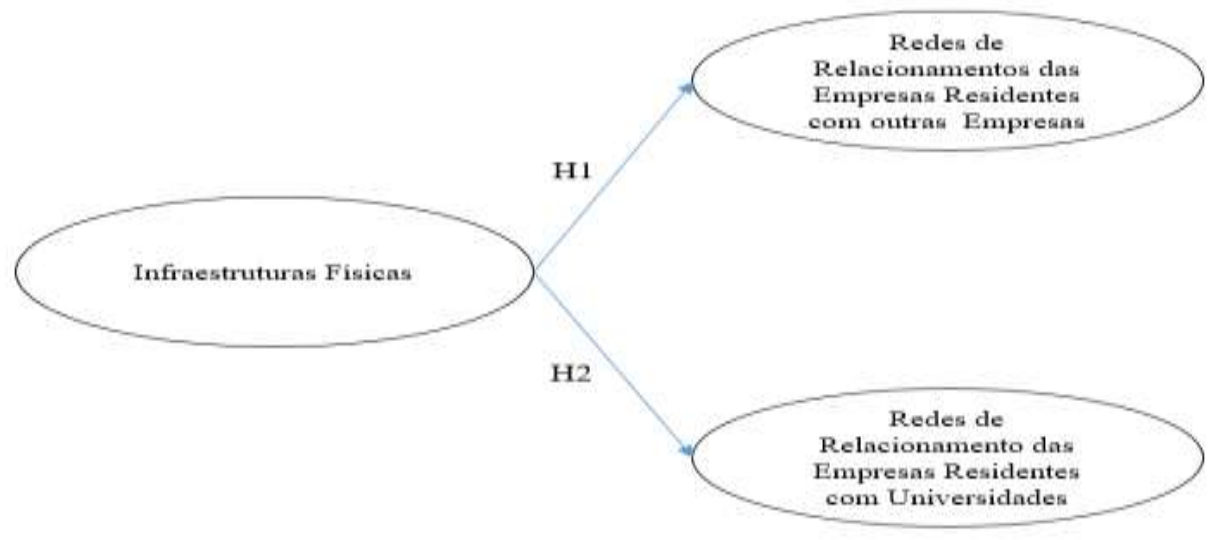

Figura 2. Modelo teórico

Fonte: Elaborado pelos autores.

\section{Procedimentos Metodológicos}

Definido o objetivo deste estudo em analisar as relações das infraestruturas físicas e as interações nas redes de relacionamentos entre EE e entre UE nos parques tecnológicos do Brasil, buscou-se, através de uma pesquisa quantitativa de cunho descritivo, um melhor entendimento do assunto no contexto dos parques tecnológicos, em que os modelos de infraestruturas físicas se tornaram uma ferramenta importante para essas interações (Atrasas et 
al., 2003; Baldoni \& Furtado, 2014; Figlioli \& Porto, 2012; Gower \& Harris, 1996) visando o estímulo a inovação (Vedovello et al., 2006).

Para alcançar este objetivo, após a realização da revisão bibliográfica para aprofundar o entendimento sobre os temas, testou-se a possível relação entre a relação da infraestrutura física (adequação) e a sua influência nas redes de relacionamento entre EE e EU nos parques tecnológicos brasileiros (ABDI \& ANPROTEC, 2008), as quais foram testadas, quantitativamente, por meio da modelagem de equações estruturais.

\subsection{Amostra}

Para verificar a relação entre as variáveis e explicá-las, optou-se pela utilização de uma pesquisa quantitativa do tipo survey. A coleta dos dados foi realizada em 2016 entre os meses de janeiro e junho. O instrumento de coleta da pesquisa foi composto de 16 itens. Após a elaboração do instrumento de coleta de dados, foram realizadas entrevistas com três pesquisadores, seis gestores do Parque Científico e Tecnológico da PUCRS (TECNOPUC) e cinco empresas residentes no parque com o objetivo de ajustá-lo à realidade das empresas. Após a realização das entrevistas, pequenos ajustes sugeridos foram realizados e aplicou-se um préteste com 34 questionários para os gestores das empresas residentes no TECNOPUC, sendo que os seus resultados foram satisfatórios e agregados à amostra final.

O questionário estruturado de autopreenchimento foi aplicado inicialmente a uma amostra de duzentos e trinta e um $(n=231)$ gestores das empresas residentes nos 22 parques tecnológicos brasileiros em operação no Brasil (parques com empresas residentes neles há no mínimo um ano), distribuídos pelas diversas regiões do país e vinculados à ANPROTEC (Associação Nacional de Entidades Promotoras de Empreendimentos Inovadores), que auxiliou na distribuição do questionário, apoiando e enviando a pesquisa por e-mail a todos os seus associados. A pesquisa foi encaminhada para 1064 empresas residentes em parques tecnológicos brasileiros.

Para participar da pesquisa, a empresa precisava ser residente há pelo menos um ano no parque, tempo considerado suficiente para o respondente conhecer e refletir sobre as infraestruturas físicas e sua relação com a interação nas redes de relacionamentos EE e UE.As empresas foram classificadas como microempresa $\left(\mathrm{n}^{\mathrm{o}}\right.$ de funcionários $\left.\leq 9\right)$, pequena empresa $\left(\mathrm{n}^{\mathrm{o}}\right.$ de funcionários $\geq 10$ e $\left.\leq 49\right)$, média empresa $\left(\mathrm{n}^{\mathrm{o}}\right.$ de funcionários $\geq 50$ e $\left.\leq 99\right)$ e grande 
empresa ( $\mathrm{n}^{\circ}$ de funcionários $>99$ ). Esta classificação foi definida pelo número de funcionários, com base nas informações do SEBRAE (Serviço Brasileiro de Apoio às Micro e Pequenas Empresas), que segue os critérios de classificação do IBGE (Instituto Brasileiro de Geografia e Estatística) para comércio e serviços (SEBRAE, 2015).

Procurando eliminar os desvios prejudiciais (casos omissos e outliers) à aplicação das técnicas estatísticas de análise do modelo teórico, foram aplicados filtros de purificação ao banco de dados. Foi empregado no questionário os procedimentos de análise de frequência das questões, observação dos casos omissos e observação dos outliers univariados e multivariados. Como resultado, nesta etapa foram eliminados 42 questionários. Após a purificação dos dados, com base na amostra válida $(n=189)$ composta por empresas residentes em 22 parques tecnológicos, espalhados pelos 12 estados brasileiros, se constatou o exposto na Figura 3, abaixo.

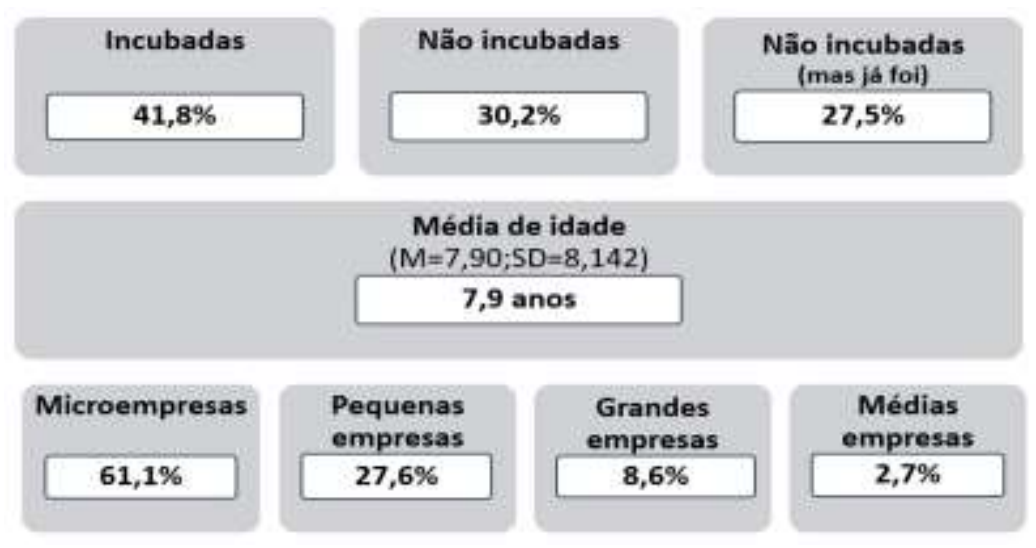

Figura 3. Perfil da amostra

Fonte: Elaborado pelos autores.

Após a coleta dos dados, foram utilizados os softwares Excel®2016, SPSS®21.0 e AMOS®21.0 para análise estatística multivariada, realizada a fim de verificar a estrutura de pesquisa proposta.

\subsection{Mensurando as variáveis}

Para realizar o teste do modelo teórico proposto na Figura 1, para mensurar as variáveis latentes, foram utilizadas escalas estabelecidas da pesquisa de Roldan (2016). A escala sobre infraestrutura física tinha como objetivo mensurar a adequação da mesma (1 - nada adequada 
e 5 - muito adequada) e as escalas utilizadas para mensurar as redes de relacionamento trabalhava com a periodicidade de uso das mesmas ( 1 - nunca e 5 - muito frequente).

Os construtos teóricos foram operacionalizados através da análise descritiva das variáveis observadas, objetivando reconhecer o comportamento das variáveis latentes. Logo após a operacionalização dos construtos, foi realizada uma análise fatorial exploratória (AFE) através do critério de Kaiser-Meyer-Olkin (KMO) e do Teste de Esfericidade de Bartlett (sig. < 0,05) com rotação Varimax, para verificar se as suposições da análise fatorial foram atendidas por cada uma das escalas de mensuração dos principais construtos.

A técnica de modelagem de equações estruturais (MEE) foi escolhida para avaliar as hipóteses desenvolvidas, verificar e corrigir erros de mensuração do processo de estimação dos construtos latentes, adotando a técnica multivariada. Buscando evitar as consequências de algumas deduções precipitadas, adotou-se a abordagem Two-step, aplicando os processos de Análise Fatorial Confirmatória (AFC), para demonstrar as relações das medidas observadas no construto e o modelo de mensuração verificando as regras de Confiabilidade Composta (CC), de Análise de Variância Média Extraída (AVE), de Validade Convergente (VC) e de Validade Discriminante (VD), para analisar a sua validade.

Logo após empregado o modelo de mensuração, aplicou-se o modelo estrutural para que dentre as múltiplas relações de dependência, fosse mostrada as associações entre os construtos do modelo e para validação, utilizando o $A M O S \AA$, se considera adequado quando se especifica o nível de significância em p $\leq$ 0,05 (Hair, Black, Babin, Anderson, \& Tatham, 2009).

\section{Apresentação e Análise dos Dados}

Visando extrair as relações indicadas na Figura 1, foi realizada uma análise multivariada dos dados, em duas etapas. Na primeira etapa foram analisados os resultados das estruturas fatorais, resultante dos processos de Análise Fatorial Exploratória da influência das infraestruturas físicas dos parques tecnológicos nas interações entre EE e UE, a partir das análises dos índices de carga fatorial $(>0,5)$, da comunalidade $(>0,5)$ e do Alfa $(\alpha)$ de cronbach; para o alfa considera-se aceitável o limite inferior de 0,60 a 0,70 (Hair et al., 2009). Na segunda etapa foram analisados os resultados de correlação, resultante da análise descritiva das variáveis 
observadas, da validade convergente e discriminante das condições favoráveis à interação em parques tecnológicos edo índice de qualidade de ajuste.

\subsection{Estruturas fatoriais}

Nesta etapa foi realizada a análise fatorial exploratória dos construtos utilizados.Para isso foi utilizado o método KMO,para cada um dos construtos estudados. A Tabela 1 apresenta os resultados globais de KMO, de Variância Extraída (VE) e alfa ( $\alpha$ de cronbach) dos construtos infraestrutura física (INFRA), redes de relacionamentos das empresas residentes com outras empresas (RELE) e redes de relacionamentos das empresas residentes com universidades (RELU), a fim de compará-los com cada uma das suas respectivas variáveis.

\section{Tabela 1}

Análise Fatorial da relação das infraestruturas físicas dos parques tecnológicos nas interações entre EE e UE.

\begin{tabular}{|c|c|c|c|c|}
\hline \multicolumn{2}{|c|}{$\begin{array}{l}\text { Infraestrutura Física } \\
\text { KMO: } 0,738 \text { VE: } 58,030 \text { Alfa: } 0,755\end{array}$} & \multirow{2}{*}{$\begin{array}{l}\text { Carga } \\
\text { fatorial } \\
0,737\end{array}$} & \multirow{2}{*}{$\begin{array}{l}\text { Com. } \\
0,542\end{array}$} & \multirow{2}{*}{$\begin{array}{l}\text { ase o item for } \\
\text { excluído } \\
0,709\end{array}$} \\
\hline INFRAI & Infraestruturas básicas & & & \\
\hline INFRA2 & Edifícios institucionais & 0,801 & 0,641 & 0,674 \\
\hline INFRA3 & Edifícios de negócios & 0,785 & 0,617 & 0,687 \\
\hline INFRA4 & Infraestruturas tecnológicas & 0,722 & 0,521 & 0,723 \\
\hline INFRA5* & Áreas verdes e sociais & 0,508 & 0,258 & 0,755 \\
\hline \multicolumn{2}{|c|}{$\begin{array}{l}\text { Redes de Relacionamento de Empresas com outras Empresas } \\
\text { KMO: } 0,785 \text { VE: } 63,353 \text { Alfa: } 0,807\end{array}$} & $\begin{array}{l}\text { Carga } \\
\text { fatorial }\end{array}$ & Com. & $\begin{array}{l}\alpha \text { se o item for } \\
\text { excluído }\end{array}$ \\
\hline RELE1 & $\begin{array}{l}\text { Combinação de ativos físicos e financeiros para a criação } \\
\text { de novas empresas }\end{array}$ & 0,782 & 0,611 & 0,767 \\
\hline RELE2 & $\begin{array}{l}\text { Uso de equipamentos, laboratórios de pesquisa e } \\
\text { instalações de outras empresas }\end{array}$ & 0,761 & 0,579 & 0,778 \\
\hline RELE3 & Parcerias de negócio & 0,802 & 0,643 & 0,755 \\
\hline RELE4 & Colaborações em projetos & 0,837 & 0,701 & 0,730 \\
\hline \multicolumn{2}{|c|}{$\begin{array}{l}\text { Redes de Relacionamento das Empresas Residentes com as } \\
\text { Universidades KMO: 0,907 VE: 63,529 Alfa: 0,917 }\end{array}$} & $\begin{array}{l}\text { Carga } \\
\text { fatorial }\end{array}$ & Com. & $\begin{array}{l}\alpha \text { se o item for } \\
\text { excluído }\end{array}$ \\
\hline RELU1 & $\begin{array}{l}\text { Aquisição de conhecimento, experiência e competências } \\
\text { da universidade através de captação de estudantes na } \\
\text { equipe }\end{array}$ & 0,712 & 0,507 & 0,914 \\
\hline RELU2 & $\begin{array}{l}\text { Aquisiçãa de conhecimento, experiência e competências } \\
\text { da universidade através de projetos de pesquisa }\end{array}$ & 0,846 & 0,715 & 0,902 \\
\hline RELU3 & Parcerias de negócio entre empresa e universidade & 0,869 & 0,755 & 0,898 \\
\hline RELU4 & Contratos de pesquisa entre empresa (s) e universidade (s) & 0,818 & 0,668 & 0,904 \\
\hline RELU5 & $\begin{array}{l}\text { Spin-out ou licenciamento de patentes das universidades } \\
\text { para exploração por parte das empresas }\end{array}$ & 0,718 & 0,515 & 0,913 \\
\hline RELU6 & $\begin{array}{l}\text { Troca de conhecimentos através de eventos na } \\
\text { universidade }\end{array}$ & 0,794 & 0,631 & 0,906 \\
\hline RELU7 & $\begin{array}{l}\text { Parcerias para o compartilhamento de conhecimento e } \\
\text { experiências }\end{array}$ & 0,829 & 0,688 & 0,903 \\
\hline RELU8 & Acesso a estruturas de pesquisa & 0,776 & 0,603 & 0,908 \\
\hline
\end{tabular}

Fonte: Dados da pesquisa 
Em relação ao construto Infraestrutura Física, a variável INFRA5* precisou ser excluída do construto, em razão do resultado da comunalidade ter ficado abaixo do indicado e a sua eliminação também elevou o $\alpha$ de Cronbach. Após a retirada do indicador INFRA5, observase que este construto se comportou adequadamente (Hair et al., 2009).

Os resultados obtidos no construto Redes de Relacionamentos entre Empresas foram considerados satisfatórios, já que todos os indicadores se comportaram adequadamente, assim como ocorreu com o construto Rede de Relacionamento entre Empresas e Universidades.

A operacionalização do construto ocorreu por meio da análise descritiva das variáveis observadas, conforme apresentado na Tabela 2.

A análise da Tabela 2 demonstra que o construto INFRA indica uma tendência positiva para as condições das infraestruturas físicas disponibilizadas pelos parques tecnológicos. Levando em consideração o resultado médio, onde a adequação predominou entre 3,54 e 3,93 indicando que a maioria dos respondentes ficou entre os pontos 3 e 4 da escala (próximo ao "muito adequado") e que o desvio padrão foi considerado baixo, indicando uma dispersão próxima da média (Hair et al., 2009). A literatura apresenta estudos onde a qualidade da infraestrutura física é considerada um item recorrente e comum (básico) para os modelos de parques tecnológicos de sucesso espalhados pelo mundo (ABDI \& ANPROTEC, 2008; Bellavista \& Sanz, 2009).

\section{Tabela 2}

Análise descritiva das variáveis observadas

\begin{tabular}{|c|c|c|c|c|}
\hline Variáveis latentes & \multicolumn{2}{|c|}{ Variáveis observadas } & Média & $\begin{array}{l}\text { Desvio } \\
\text { padrão }\end{array}$ \\
\hline \multirow{4}{*}{$\begin{array}{l}\text { Infraestrutura } \\
\text { Física }\end{array}$} & INFRA1 & Infraestruturas básicas & 3,76 & 1,00 \\
\hline & INFRA2 & Edifícios institucionais & 3,93 & 1,01 \\
\hline & INFRA3 & Edifícios de negócios & 3,82 & 0,94 \\
\hline & INFRA4 & Infraestruturas tecnológicas & 3,54 & 1,11 \\
\hline \multirow{4}{*}{$\begin{array}{l}\text { Redes de } \\
\text { Relacionamento } \\
\text { entre empresas }\end{array}$} & RELE1 & $\begin{array}{l}\text { Combinação de ativos físicos e financeiros para a criação } \\
\text { de novas empresas }\end{array}$ & 2,34 & 1,15 \\
\hline & RELE2 & $\begin{array}{l}\text { Uso de equipamentos, laboratórios de pesquisa e } \\
\text { instalações de outras empresas }\end{array}$ & 2,27 & 1,13 \\
\hline & RELE3 & Parcerias de negócio & 3,05 & 1,17 \\
\hline & RELE4 & Colaborações em projetos & 2,81 & 1,19 \\
\hline \multirow{4}{*}{$\begin{array}{l}\text { Redes de } \\
\text { Relacionamento } \\
\text { entre empresas e } \\
\text { universidades }\end{array}$} & RELU1 & $\begin{array}{l}\text { Aquisição de conhecimento, experiência e competências } \\
\text { da universidade através de captação de estudantes na } \\
\text { equipe }\end{array}$ & 3,14 & 1,27 \\
\hline & RELU2 & $\begin{array}{l}\text { Aquisição de conhecimento, experiência e competências } \\
\text { da universidade através de projetos de pesquisa }\end{array}$ & 3,00 & 1,22 \\
\hline & RELU3 & Parcerias de negócio entre empresa e universidade & 2,93 & 1,37 \\
\hline & RELU4 & Contratos de pesquisa entre empresa (s) e universidade (s) & 2,67 & 1,26 \\
\hline
\end{tabular}




\begin{tabular}{|l|l|l|l|l}
\hline & RELU5 & $\begin{array}{l}\text { Spin-out ou licenciamento de patentes das universidades } \\
\text { para exploração por parte das empresas }\end{array}$ & 2,18 & 1,09 \\
\cline { 2 - 6 } & RELU6 & $\begin{array}{l}\text { Troca de conhecimentos através de eventos na } \\
\text { universidade }\end{array}$ & 3,23 & 1,23 \\
\cline { 2 - 6 } & RELU7 & $\begin{array}{l}\text { Parcerias para o compartilhamento de conhecimento e } \\
\text { experiências }\end{array}$ & 3,06 & 1,19 \\
\cline { 2 - 6 } & RELU8 & Acesso a estruturas de pesquisa & 2,89 & 1,35 \\
\hline
\end{tabular}

Fonte: Dados da pesquisa

Quando se trata de infraestrutura física nos parques são consideradas adequadas pelas empresas respondentes, em destaque os edifícios institucionais que abrigam a organização gestora do parque, associações representativas das empresas, bancos de fomento e agências de desenvolvimento. Nesse sentido, os parques brasileiros atendem às diretrizes conceituais estabelecidas pela Associação Nacional de Entidades Promotoras de Empreendimentos Inovadores (2006), quando relacionado um complexo produtivo industrial e de serviços de base científico-tecnológica planejado, e de acordo com Vedovello, Judice e Maculan (2006), como um local delimitado e de infraestrutura adequando aos diferentes negócios que se pretende empreender. A particularidade, no Brasil, como aponta Vedovello, Judice e Maculan (2006), demonstra que parques tecnológicos têm sido concebidos prioritariamente à implementação de infraestrutura física de apoio às empresas, negligenciado aspectos intangíveis importantes para o processo de inovação e o fortalecimento da interação empresa-empresa e empresauniversidade, como é possível observar nas variáveis que avaliam as redes de relacionamento EE e EU.

Para o construto RELE, o resultado mostra que a frequência das interações EE tende a ser baixa, em razão da média ter ficado entre 2,27 e 3,05 com 75\% dos respondentes ficando abaixo do ponto 3 da escala (tendendo ao penúltimo valor mínimo da escala), com um desvio padrão baixo, indicando que a dispersão se mantém próxima a média (Hair et al., 2009). A média do construto RELE está baixa e provavelmente está correlacionado ao baixo grau de inovação das empresas brasileiras e o receio de se relacionar por serem consideradas possíveis concorrentes e que possam possuir alguma vantagem que pode ser prejudicial para uma das empresas. Porém, nas empresas incubadas no Daresbury SIC, a frequência de relacionamento também apresentou um resultado baixo para as empresas de cunho tecnológico (Soetanto \& Jack, 2013).

A empresa é considerada o lócus da produção e a principal usuária da inovação (Etzkowitz, 2013). Em países emergentes, a interação de empresas é uma alternativa para complementar seus recursos geralmente limitados (Zawislak \& Dalmarco, 2011). O 
estabelecimento de uma colaboração equilibrada entre tecnologia e negócios, segundo Etzkowitz (2013), é um elemento determinante para a criação de uma startup com significado potencial de crescimento. A baixa interação entre empresas pode representar um obstáculo à inovação que, apesar de estarem localizadas em parque tecnológico, ainda há um longo caminho a percorrer.

Os resultados também demonstram que a inovação aberta proposta por Chesbrough (2012), embora não seja um elemento analisado no presente artigo, ainda não é um modelo utilizado pelas empresas, como alternativa ao modelo tradicional de gestão, nos parques brasileiros, em especial à variável analisada "uso de equipamentos, laboratórios de pesquisa e instalações de outras empresas", de menor média atribuída. Recursos internos e externos à empresa podem ser explorados simultaneamente pela adoção do processo de gestão aberta, contribuindo assim para melhores resultados de interação nas redes de relacionamento EE.

Já para o construto RELU, o resultado demonstrou neutralidade referente à interação UE, onde a média predominou no centro da escala (entre 2,18 e 3,23), ficando $50 \%$ dos respondentes acima (entre 3,00 e 3,23) e os outros a abaixo (entre 2,18 e 2,93), com um desvio padrão baixo, indicando que a dispersão se mantém próxima a média (Hair et al., 2009). Provavelmente este resultado ocorreu devido à baixa interação existente ainda entre empresas e universidades, assim, a cultura de interação entre estes atores ainda não está consolidada. Em decorrência de uma baixa interação, ocasionalmente as pesquisas realizadas nas universidades podem ser pouco atrativas para a transferência de conhecimento, onde o sucesso dos processos de interação entre UE se evidencia quando os resultados das pesquisasse tornam úteis, e assim, são transferidos às empresas. Desta forma pode-se justificar o resultado de neutralidade em razão de pesquisas que não apresentam os resultados que sejam atrativos para a indústria (Audy et al., 2002).

Estudos têm demonstrado que a interação universidade e empresa no Brasil ainda é modesta (Fernandes et al., 2010; Suzigan \& Albuquerque, 2008; Vedovello et al., 2006). Apesar do recente e crescente estímulo de políticas de ciência, tecnologia e inovação para incentivar à pesquisa cooperada e a inserção de pesquisadores em empresas, os principais motivos que levam as empresas interagirem ainda são de interesses particulares, como a contratação de recursos humanos, alunos, para acesso a novos conhecimentos, e acesso à infraestrutura oferecida pela universidade (Gielfi et al., 2017). 


\subsection{Análise de correlação e modelo estrutural}

Nesta etapa foram analisados os resultados de correlação, através dos testes de validade convergente (VC) e discriminante (VD) e do modelo estrutural, todos por meio do modelo de mensuração da modelagem de equações estruturais. Para análise dos testes de VC e VD foram utilizados os indicadores de CC e da AVE dos fatores. Para os resultados de validade, foi empregado o critério de Fornell e Larcker (1981), a fim de comprovar a correlação entre as variáveis estudadas.

Analisados os resultados dos testes observou-se que os índices do teste de confiabilidade composta mostram-se satisfatórios, uma vez que os valores dos construtos (INFRA, RELE e RELU $\geq 0,7$ ) ficaram acima do indicado pela literatura (Hair et al., 2009). Para o teste da variância média extraída também se mostrou adequado, devido aos índices dos construtos (INFRA, RELE e RELU $\geq 0,5$ ) terem ficado acima do indicado, confirmando validade convergente para as medidas deste modelo (Hair et al., 2009). Para o teste de validade discriminante o modelo se mostrou fidedigno uma vez que o valor da raiz quadrada da AVE foi superior aos valores das correlações entre os construtos (Fornell \& Larcker, 1981).

A Tabela 2 mostra os dados do teste de ajustamento global do modelo, a magnitude dos coeficientes de regressão e o seu nível de significância, executado através da ferramenta AMOSTM.

\section{Tabela 3}

Índice de Qualidade de Ajustamento final

\begin{tabular}{|c|c|c|c|c|}
\hline Medidas & Estatística & Construto & Valor de referência & Referência \\
\hline Absoluta & $\begin{array}{l}\mathrm{x}^{2} \text { e p-value } \\
\mathrm{x}^{2} / \mathrm{gl} \\
\text { RMSEA }\end{array}$ & $\begin{array}{l}161,808 \\
1,634 \\
0,058\end{array}$ & $\begin{array}{l}\text { Quanto menor, melhor e } \mathrm{p}>0,05 \\
\leq 5 \\
\leq 0,08\end{array}$ & \\
\hline Comparativa & $\begin{array}{l}\text { GFI } \\
\text { AGFI } \\
\text { TLI } \\
\text { NFI } \\
\text { CFI }\end{array}$ & $\begin{array}{l}0,903 \\
0,867 \\
0,950 \\
0,901 \\
0,959\end{array}$ & $\begin{array}{l}\geq 0,9 \\
\geq 0,9 \\
\geq 0,9 \\
\geq 0,9 \\
\geq 0,9\end{array}$ & Hair et al. (2009) \\
\hline
\end{tabular}

Fonte: Elaborado pelos autores.

Analisando o construto do modelo estrutural final observa-se que a adequação atende os preceitos da literatura de modelagem, com exceção do indicador absolut good fit index $(\mathrm{AGFI}=0,867)$ que não apresentou um resultado satisfatório, mas tendo em vista que o índice ficou muito próximo do indicado pela literatura, acredita-se que não afetará o resultado do 
ajustamento. Sendo assim pode-se considerar que o modelo estrutural do ajustamento final se apresentou adequado. A Figura 3 abaixo ilustra o modelo estrutural final do construto.

Com o modelo estrutural final ajustado e adequado, a próxima etapa foi efetuar os testes de hipóteses, a partir da premissa do modelo teórico inicial sobre a relação da adequação das infraestruturas físicas dos parques tecnológicos nas interações entre EE e UE, onde a infraestrutura física dos parques deveria influenciar positivamente nas interações das redes de relacionamento entre as empresas residentes $(\mathrm{H} 1)$ e entre as empresas e a universidade $(\mathrm{H} 2)$, conforme indicado na Figura 4:

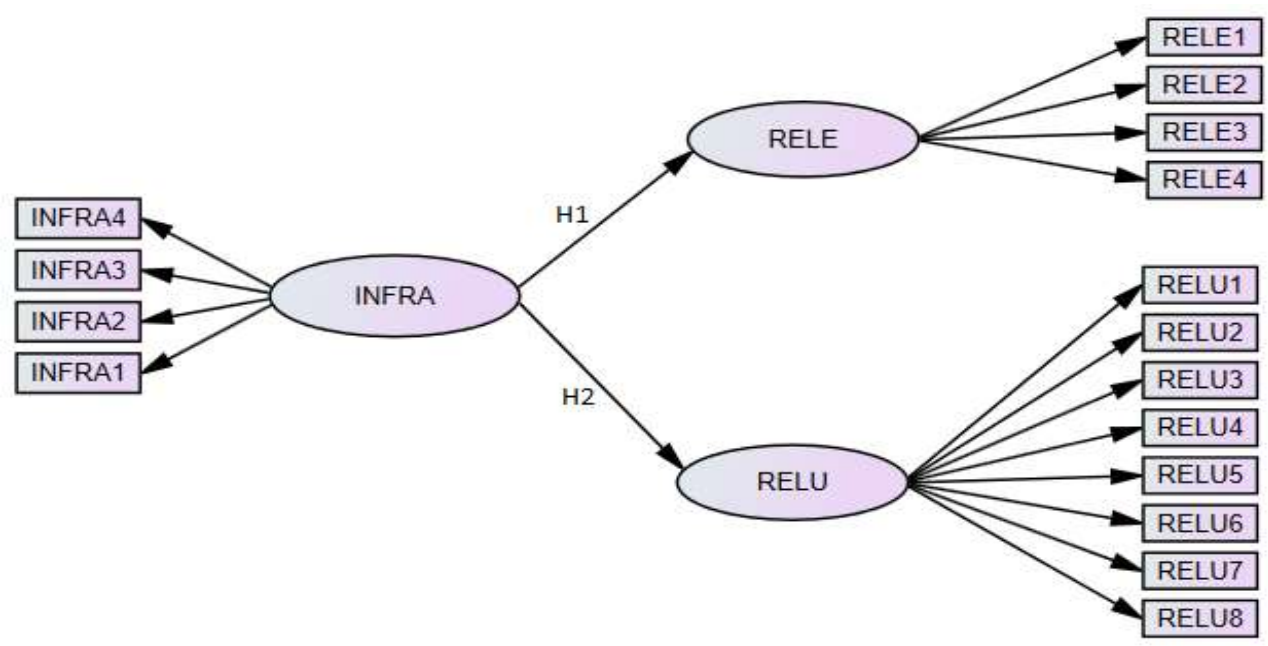

Figura 4: Modelo estrutural final Fonte: Elaborado pelos autores.

A Tabela 3 mostra o teste de hipóteses do modelo de mensuração da relação da adequação das infraestruturas físicas nas interações nas redes de relacionamentos entre EE e EU das hipóteses do construto, onde serão analisados os resultados de Beta $(\beta)$, z-test e p-value para aceitar ou rejeitar cada hipótese.

\section{Tabela 4}

Teste de Hipóteses do Modelo

\begin{tabular}{l|l|l|l|l|l|l|l|l}
\hline \multicolumn{2}{l}{ Hipóteses } & B & Erro Padronizado & z-test & p-value & Teste \\
\hline H1 & RELE & $\leftarrow$ & AINFRA & 0,369 & 0,128 & 3,651 & $* * *$ & Aceita \\
\hline H2 & RELU & $\leftarrow$ & AINFRA & 0,399 & 0,135 & 4,083 & $* * *$ & Aceita \\
\hline
\end{tabular}

Nota: $* * *$ p-value $<0,001$

Fonte: Elaborado pelos autores. 
Em análise a hipótese de que as infraestruturas físicas dos parques tecnológicos influenciam nas redes de relacionamentos das empresas residentes com outras empresas $(\mathrm{H} 1)$ ocorreu uma relação positiva, onde foram encontrados os resultados para $\beta(=0,369)$, z-test (= $3,651)$ e para $p$-value $(=0,000)$. Este resultado demonstra que as infraestruturas físicas dos parques tecnológicos influenciam positivamente nas interações nas redes de relacionamentos entre EE. Este resultado pode ser confirmado na literatura através dos estudos de Soetanto e Jack (2013) e Cantù (2010) onde se percebe a relação da infraestrutura física com a interação entre as empresas.

Em análise a hipótese de que as infraestruturas físicas dos parques tecnológicos influenciam nas redes de relacionamentos das empresas residentes com a universidade $(\mathrm{H} 2)$ também ocorreu uma relação positiva, onde foram encontrados os resultados para $\beta(=0,399)$, z-test $(=4,083)$ e para p-value $(=0,000)$. Este resultado demonstra que as infraestruturas físicas dos parques tecnológicos também influenciam positivamente nas interações nas redes de relacionamentos entre UE, e pode ser percebido na literatura através de alguns estudos (Audy et al., 2002; Cantù, 2010).

\section{Conclusões e Recomendações}

Buscando responder à questão da pesquisa, este estudo estudou a relação da infraestrutura física dos parques tecnológicos com as interações nas redes de relacionamento entre as empresas residentes (H1) e entre as empresas e as universidades (H2). Depois de realizada a revisão bibliográfica, foi utilizada uma amostra de 189 empresas instaladas em parques tecnológicos no Brasil para aplicação da pesquisa. Um modelo teórico foi criado e testado quantitativamente, através da modelagem de equações estruturais, onde se buscou analisar as relações propostas.

Os resultados das análises estatísticas possibilitam presumir que há influência da qualidade da infraestrutura física nas interações das redes de relacionamentos entre as empresas residentes e entre as empresas e as universidades dentro dos parques tecnológicos estudados.

Os resultados obtidos neste estudo mostram a importância da infraestrutura física dos parques tecnológicos, sugerindo que para se obter uma rede de relacionamento atuante se faz necessário uma infraestrutura física capaz de gerar interação. Neste contexto, a literatura mostra que a qualidade da infraestrutura física é um ponto importante nas interações (ABDI \& ANPROTEC, 2008; Bellavista \& Sanz, 2009). Sendo assim, um aprofundamento nesta questão 
através de um estudo qualitativo, poderia auxiliar os gestores dos parques a encontrar a melhor configuração destas infraestruturas, a fim de melhorar a quantidade e a frequência destas interações, uma vez que nem todos os parques tecnológicos apresentam condições adequadas de infraestrutura.

Levando em consideração que hoje as empresas não conseguem mais serem competitivas sem parcerias (Molina-Morales \& Martínez-Cháfer, 2016), o efeito de aglomeração e proximidade mostra-se um fator básico para o desenvolvimento dos parques tecnológicos na busca do desenvolvimento das empresas, do desenvolvimento local e regional (Porter, 1999). Já que a literatura aborda que as interações nas redes de relacionamentos dos parques tecnológicos são importantes para o desenvolvimento e crescimento das empresas e, por sua vez, este estudo mostra que a adequação da infraestrutura física é capaz de influenciar as interações nestas redes, sugere-se para estudos futuros um levantamento das infraestruturas consideradas mais adequadas para esta finalidade, a fim de potencializar as interações em busca de benefícios mútuos para os parques, empresas e universidades. Pode-se sugerir também um estudo qualitativo focado nas razões pelas quais as infraestruturas físicas são consideradas uma fomentadora de interações.

Como contribuição teórica, este estudo ajudou a demonstrar que não basta a existência de infraestruturas físicas nos parques tecnológicos; estas precisam ser adequadas ao ambiente para que gerem interações entre as empresas e entre empresas e universidades. Assim, evidencia-se que os governos precisam fomentar não apenas a criação de parques tecnológicos, mas sim de parques tecnológicos que possuam infraestruturas físicas capazes de proporcionar interação entre os atores do sistema de inovação. Uma questão a ser melhor analisada é a diferença de importância das condições de infraestrutura adequadas para os diferentes setores ou atividades econômicas inseridas nos parques.

A pesquisa realizada também se mostra relevante, ao considerar o contexto dos parques tecnológicos no Brasil de uma forma ampla (todos os parques tecnológicos brasileiros em operação), de acordo com a bibliografia considerada. Porém, estes resultados devem ser visualizados a partir das empresas residentes em parques tecnológicos brasileiros, não podendo ser generalizados para contextos amostrais muito distintos deste. Com o objetivo de superar esta limitação, sugere-se que novas coletas de dados sejam realizadas com outras amostras, em outros contextos e em outros países, possibilitando comparações futuras entre os resultados. 


\section{Referências}

ABDI, \& ANPROTEC. (2008). Parques Tecnológicos no Brasil: Estudo, Análise e Proposições. Angeloni, M. T. (2002). Organizações do Conhecimento: Infraestrutura, pessoas e tecnologias. São Paulo: Editora Saraiva.

Araujo, L., Dubois, A., \& Gadde, L. (2003). The Multiple Boundaries of the Firm*. Journal of Management Studies, (July), 1255-1275.

Atrasas, A. L., Dias, J. M. C. de S., \& Leite, L. A. de S. (2003). PARQUES TECNOLÓGICOS E INCUBADORAS DE EMPRESAS: FATORES DE DESENVOLVIMENTO TECNOLÓGICO E REGIONAL EM PORTUGAL E ESPANHA: Relatório de viagem internacional. Embrapa - Recursos Genéticos E Biotecnologia. Brasília.

Audy, J. L. N., Cunha, N. C. V. da, \& Franco, P. R. G. (2002). TECNOPUC: UMA PROPOSTA DE HABITAT DE INOVAÇÃO PARA PORTO ALEGRE. In ANPROTEC (p. 15).

Baldoni, L., \& Furtado, A. T. (2014). Parque Científico e Tecnológico da Unicamp e seu Entorno: Quais perpectivas para consolidar um Ambiente de Inovação ? (ANPROTEC, Ed.), XXIV Seminário Nacional de Parques Tecnológicos E Incubadoras de Empresas. Bélem.

Bellavista, J., \& Sanz, L. (2009). Science and technology parks: habitats of innovation: introduction to special section. Science and Public Policy, 36(August), 499-510. https://doi.org/10.3152/030234209X465543

Cantù, C. (2010). Exploring the role of spatial relationships to transform knowledge in a business idea - Beyond a geographic proximity. Industrial Marketing Management, 39(6), 887-897. https://doi.org/10.1016/j.indmarman.2010.06.008

Etzkowitz, H. (2013). Hélice Tríplice: Universidade-Industria-Governo: Inovação em Movimento (1st ed.). Porto Alegre: EDIPUCRS.

Etzkowitz, H., \& Zhou, C. (2018). Innovation incommensurability and the science park. $R \& D$ Management, 48(1), 73-87.

Fernandes, A. C., de Souza, B. C., Da Silva, A. S., Suzigan, W., Chaves, C. V., \& Albuquerque, E. (2010). Academy-industry links in Brazil: Evidence about channels and benefits for firms and researchers. Science and Public Policy, 37(7), 485-498. https://doi.org/10.3152/030234210X512016

Ferreira, G. C., Soria, A. F., \& Closs, L. (2012). Gestão da interação Universidade-Empresa: o caso PUCRS. Revista Sociedade E Estado, 27(1), 79-95.

Figlioli, A., \& Porto, G. S. (2012). Financiamento de parques tecnológicos: um estudo comparativo de casos brasileiros, portugueses e espanhóis. Revista de Administração, 47, 290-306. https://doi.org/10.5700/rausp1040

Fornell, C., \& Larcker, D. F. (1981). Evaluating Structural Equation Models with Unobservable Variables and Measurement Error. Journal of Marketing Research (JMR). Feb1981, 18(1), 39-50. 12p. 1 Diagram. https://doi.org/10.2307/3151312

Gower, S., \& Harris, F. (1996). Evaluating British science parks as property investment opportunities. Journal of Property Valuation and Investment, 14(2), 24-37. https://doi.org/10.1108/14635789610112646

Hair, J. F., Black, W. C., Babin, B. J., Anderson, R. E., \& Tatham, R. L. (2009). Análise Multivariada de Dados (6th ed.). Porto Alegre: Bookman.

Minguillo, D., Tijssen, R., \& Thelwall, M. (2015). Do science parks promote research and technology? A scientometric analysis of the UK. Scientometrics, 102(1), 701-725. https://doi.org/10.1007/s11192-014-1435-z

Molina-Morales, F. X., \& Martínez-Cháfer, L. (2016). Cluster Firms: You'll Never Walk Alone. Regional Studies, 50(5), 877-893. https://doi.org/10.1080/00343404.2014.952719

Porter, M. (1999). A vantagem competitiva das nações. Competição: Estratégias Competitivas 
Essenciais.

Pradella, S. (2013). OS PARQUES CIENTÍFICOS E TECNOLÓGICOS E A INOVAÇÃO: Reflexos no desenvolvimento local. XV Encontro Da Anpur, Recife. XV ENanpur Desenvolvimento, Planejamento E Governança, 15, 17.

Roldan, L. B. (2016). Análise das Condições Favoráveis para a Inovação e seus Resultados nas Empresas Residentes em Parques Tecnológicos. Pontifícia Universidade Católica do Rio Grande do Sul. Retrieved from http://tede2.pucrs.br/tede2/bitstream/tede/7082/2/TES_LUCAS_BONACINA_ROLDAN_C OMPLETO.pdf

SEBRAE. (2015). Anuário do Trabalho na Micro e Pequena Empresa: 2014. Sistema Brasileiro de Apoio às Micro e Pequenas Empresas/Departamento Intersindical de Estatística e Estudos Socioeconômicos. São Paulo. https://doi.org/1983-2095

Sereda, L., Dietrich, P. M., \& Hermann, T. A. (2015). Indicadores de desempenho como suporte estratégico para acompanhamento de projetos de inovação e tecnologia. Revista Brasileira de Gerenciamento de Projetos - RBGP, 16(3), 5-9.

Soetanto, D. P., \& Jack, S. L. (2013). Business incubators and the networks of technology-based firms. Journal of Technology Transfer, 38, 432-453. https://doi.org/10.1007/s10961-0119237-4

Spolidoro, R., \& Audy, J. (2008). Parque Científico e Tecnológicos da PUCRS. Porto Alegre: ediPUCRS.

Suzigan, W., \& Albuquerque, E. D. M. E. (2008). A Interação entre universidades e empresas em perspectiva histórica no Brasil. Texto Para Discussão, (329), 1-27.

Vedovello, C. A., Judice, V. M. M., \& Maculan, A.-M. D. (2006). Revisão crítica às abordagens a parques tecnológicos: alternativas interpretativas às experiências brasileiras recentes. $R A I$ : $\begin{array}{lllll}\text { Revista de Administração } & \text { Inovação, } & 3, & 103-118 .\end{array}$ https://doi.org/http://dx.doi.org/10.5585/rai.v3i2.58

Wolfarth, C. P. (2004). Parques tecnológicos : uma proposta de modelo de gestão a partir do estudo de caso do Pólo de Informática de São Leopoldo. Universidade Federal do Rio Grande do Sul. Retrieved from http://www.lume.ufrgs.br/handle/10183/6460

Zawislak, P. A., \& Dalmarco, G. (2011). The silent run: New issues and outcomes for Universityindustry relations in Brazil. Journal of Technology Management and Innovation, 6(2), 66-82. https://doi.org/10.4067/S0718-27242011000200005 